\title{
Absence of Expression of OCT-2 in Gastric Cancer Cells
}

\author{
José Paulo Freire ${ }^{*}$, Maria Emilia Oliveira², Maria Conceição Crujo², Ana Rute Fernandes², \\ José Crespo Mendes de Almeida ${ }^{1}$, Henrique Bicha Castelo ${ }^{1}$ \\ ${ }^{1}$ Department of Surgery, Hospital de Santa Maria, Faculdade de Medicina de Lisboa, Lisboa, Portugal \\ ${ }^{2}$ Department of Pathology, Hospital de Santa Maria, Faculdade de Medicina de Lisboa, Lisboa, Portugal \\ Email: ${ }^{*}$ freire20@sapo.pt
}

Received 13 April 2014; revised 12 May 2014; accepted 11 June 2014

Copyright (C) 2014 by authors and Scientific Research Publishing Inc.

This work is licensed under the Creative Commons Attribution International License (CC BY).

http://creativecommons.org/licenses/by/4.0/

(c) (i) 0pen Access

\begin{abstract}
OCT-2, a member of the POU homeodomain family of transcription factors, has been implicated in gastric cancer lymph node metastasis on a single publication so far. Other members of the same family of transcription factors were found to have a role in intestinal metaplasia and gastric cancer (OCT-1) and in trophectoderm differentiation of embryonic stem cells (OCT3/4) via modulation of CDX2. These findings prompted us to evaluate whether OCT-2 could in fact have a role in gastric oncogenesis and lymph node metastasis in human gastric cancer, designing an immunohistochemistry study of the putative expression of OCT-2 on human gastric cancer tissue of 69 surgical archival specimens of intestinal and diffuse type. We could not find expression of OCT-2 on any of our cancer tissues, metaplasia or normal gastric mucosa despite the expression of OCT-2 on tonsil lymphatic cells (tissue test) and normal lymph nodes. We concluded that, based on immunohistochemistry, OCT-2 does not have a role in gastric oncogenesis nor does have any relationship with lymph node metastasis in gastric cancer.
\end{abstract}

\section{Keywords}

Gastric Cancer, Lymph Node Metastasis, OCT-2, Stem Cells

\section{Introduction}

OCT-2 is a member of the POU homeodomain family of transcription factors [1] and was originally identified as a factor which was expressed only in B Lymphocytes. Subsequently, the OCT-2 factor was also identified in neuronal cells [2]. OCT-2 protein is encoded by a single gene but the primary transcript can be spliced in a number of different ways to yield different protein variants with different activities. It has been shown that his alternative splicing is regulated differently in different cell types and that it may play an important role in the

\footnotetext{
"Corresponding author.
}

How to cite this paper: Freire, J.P., Oliveira, M.E., Crujo, M.C., Fernandes, A.R., de Almeida, J.C.M. and Castelo, H.B. (2014) Absence of Expression of OCT-2 in Gastric Cancer Cells. American Journal of Molecular Biology, 4, 129-133. 
normal development of mammals [3].

Recently, it was demonstrated that OCT-1, another member of the POU homeodomain family of transcription factors that share with OCT-2, the ability to recognize the canonical octamer motif (ATGCAAAT) that regulates the transcription of various genes, is over expressed in intestinal metaplasia and intestinal type carcinomas in human gastric cancer [4]. Also some experiments with high-density oligonucleotide microarray analysis suggested that OCT-2 could be overexpressed in human gastric cancer with a significant statistical relationship with lymph node metastasis [5].

These observations prompted us to evaluate whether OCT-2 could in fact have a role in lymph node metastasis in human gastric cancer.

\section{Tissue Samples and Population Data}

For the study of OCT-2 expression in human gastric cancer we set on using immuno histochemistry essay on 69 paraffin-embedded samples from 69 gastric carcinomas obtained from patients undergoing gastrectomy in Department of Surgery of the Hospital Santa Maria, Lisboa, Portugal (Table 1). These cancer cases are all fully documented for pathological and clinical staging. According with Lauren histological classification, 20 gastric cancers were of diffuse type and 49 were of intestinal type. Follow-up was in excess of 5 years. All were operated on with either a D1 or D2 lymph node dissection (16 to 25 lymph or 26 or more nodes harvested, respectively), and thus a correct lymph node staging was available according with the AJCC/UICC 2002 edition (N0: 0 lymph node metastasis; N1: 1 to 6 lymph node metastasis; N2: 7 to 15 lymph node metastasis and N3: >16 Lymph node metastasis). Twenty four gastric cancers had no lymph node metastases (N0) and 45 presented with lymph node metastases $(\mathrm{N}+)$. Table 1 summarizes the data concerning the population being studied. Kaplan Meier life table analyzes were available in case OCT-2 expression was detected and results justified statistical analyzes.

\section{Materials and Methods}

Sections of $2 \mu \mathrm{m}$ were cut from 69 appropriately selected archival paraffin blocks (material fixed in buffered formalin and embedded in paraffin). These were mounted on SuperFrost ${ }^{\circledR}$ Plus glass slides and dried at $80^{\circ} \mathrm{C}$ for 15 minutes.

The sections were deparaffinised and hydrated. The endogenous peroxidase activity was blocked incubating the sections in a 1.5\% Methanol solution with Hydrogen Peroxide for 10 minutes.

Antigen unmasking was carried out in a microwave oven using a buffered Boric Acid solution pH 7.0, $0.02 \mathrm{M}$ (Sigma Aldrich).

Sections were incubated with rabbit polyclonal anti-Oct-2 (C-20) antibody (ref. Sc-233, Santa Cruz Biotechnology) at 1:500 in a humid chamber for 30 minutes at room temperature. The positive and negative controls were made in normal tonsil and lymph nodes. Negative control was obtained by the absence of the primary antibody. Between incubations the slides were rinsed with a $0.04 \%$ phosphate-buffered saline (PBS, Biochrom AG) solution with Triton-X100. Signal amplification and detection was made with Dako REALTM EnVisionTM Detection System, Peroxidase/DAB+, Rabbit/Mouse (code K5007, Dako) for 25 minutes. DAB solution (3,3'diaminobenzidine tetrahydrochloride) was used as the chromogen. The slides were counterstained with haematoxylin, dehydrated in increasing grades of ethanol, cleared in xylene and mounted in Entellan ${ }^{\circledR}$.

Nuclear staining was to expect, according to Santa Cruz data sheet and results with tissue test (tonsil lymphatic cells). It was decided, at first, that staining will be classified as negative if no staining was identified, weak (and also negative) if staining was clearly inferior to positive control, and positive only if staining was identical or slightly inferior to positive control. We also decided arbitrarily, that a least $10 \%$ of gastric cancer cells should stain positive in order to classify the tumor as expressing OCT-2. We were prepared to review these criteria according with the different aspects to be seen during the study and for reasons that will be apparent below we tried a different OCT-2 vial from Santa Cruz and a different immunohistochemistry protocol on 35 of the above mentioned cancer tissue samples.

\section{Statistical Analysis}

Statistical analysis would be performed in order to evaluate association of the expression of OCT-2 with: the 
Table 1. Population general data.

\begin{tabular}{|c|c|c|}
\hline Age range & $30-85$ & \\
\hline Sex distribution & Male 44 & Female 25 \\
\hline \multirow[t]{2}{*}{ Gastric cancer type } & Intestinal & 49 \\
\hline & Diffuse & 20 \\
\hline \multirow[t]{3}{*}{ Cancer localization } & Proximal third & 12 \\
\hline & Medial third & 20 \\
\hline & Distal third & 37 \\
\hline \multirow[t]{2}{*}{ Lymphadenectomy } & D1 (16 to 25 nodes harvested) & 47 \\
\hline & D2 (more then 26 nodes harvested) & 22 \\
\hline \multirow[t]{4}{*}{ T classification } & $\mathrm{T} 1$ & 10 \\
\hline & $\mathrm{T} 2$ & 24 \\
\hline & T3 & 33 \\
\hline & $\mathrm{T} 4$ & 2 \\
\hline \multirow[t]{2}{*}{$\mathrm{N}$ stage } & No & 24 \\
\hline & $\mathrm{N}+(1$ or more lymph node metastases $)$ & 45 \\
\hline \multicolumn{3}{|l|}{ TNM stage } \\
\hline & IA & 7 \\
\hline & IB & 14 \\
\hline & II & 9 \\
\hline & IIIA & 12 \\
\hline & IIIB & 7 \\
\hline & IV & 20 \\
\hline
\end{tabular}

Gastric cancer type, the T classification, the $\mathrm{N}$ and the TNM stages, and other population data. For this, it would be used the chi-square test and the differences with a p-value $\leq 0.01$, would be considered statistically significant.

The Kaplan-Meier method would be performed to estimate the survival curve for these patients and in order to evaluate the effect of the expression of OCT-2 on the survival rate, the Logrank test would be used to compare groups. Here also, only the p-value $\leq 0.01$ would be considered statistically significant.

\section{Histological Findings and Results}

Although a significant unequivocal expression of OCT-2 was found in normal tonsil and lymph nodes on positive controls as expected, none of our cancer samples stained positive for OCT-2, either intestinal or diffuse type gastric cancer (Figure 1 and Figure 2), with or without lymph node metastasis. On occasions, lymph nodes near the carcinoma tissue stained positive for OCT-2 but no staining was present in cancer tissue, metaplasia or normal gastric mucosa (Figure 3). We even tried a different OCT-2 vial from Santa Cruz and a different immunohistochemistry protocol on 35 of our cases of cancer tissue samples but no staining was identified. We can therefore conclude with confidence that gastric cancer, either diffuse or intestinal type, do not express OCT-2. We can also conclude that normal gastric epithelia and metaplastic or dysplasic gastric epithelia do not express OCT-2.

\section{Discussion}

OCT-2 is a member of the POU homeodomain family of transcription factors, originally identified as a factor which was expressed in B Lymphocytes and subsequently identified in neuronal cells. OCT-2 protein is encoded 


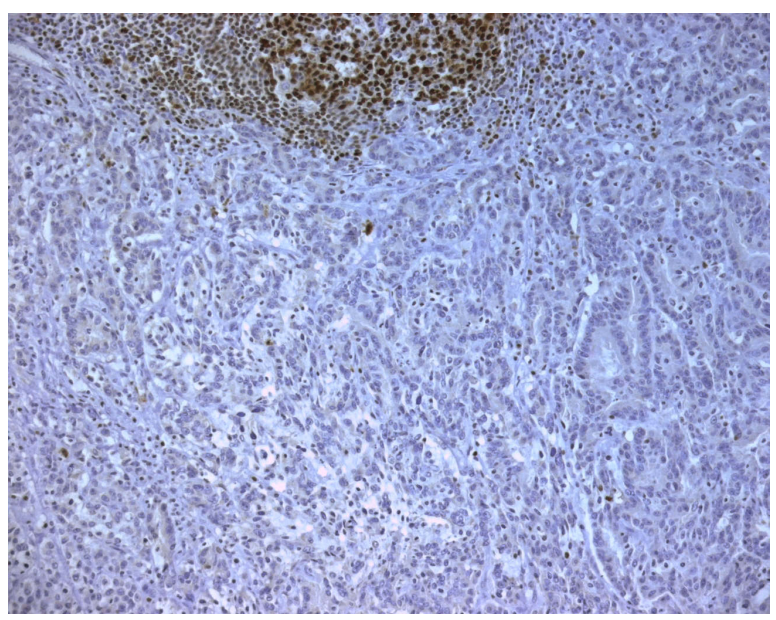

Figure 1. Intestinal gastric cancer.

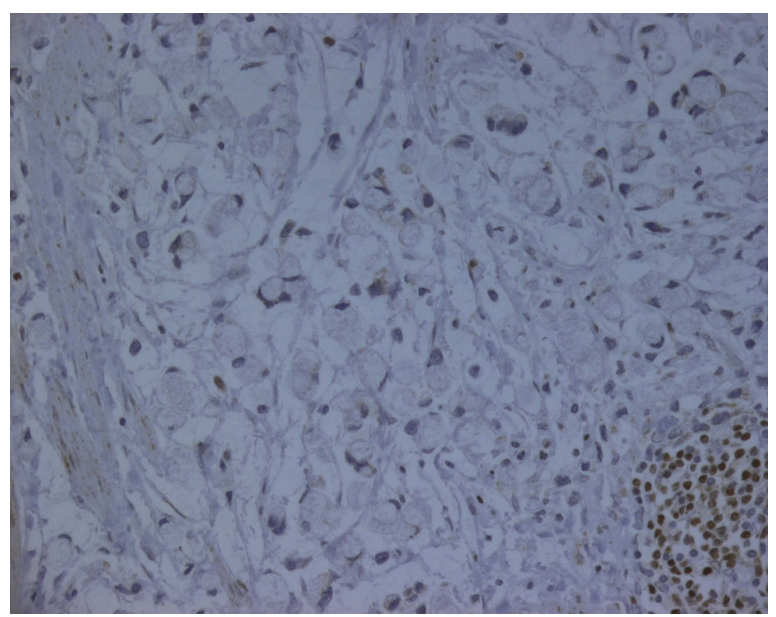

Figure 2. Diffuse type gastric cancer.

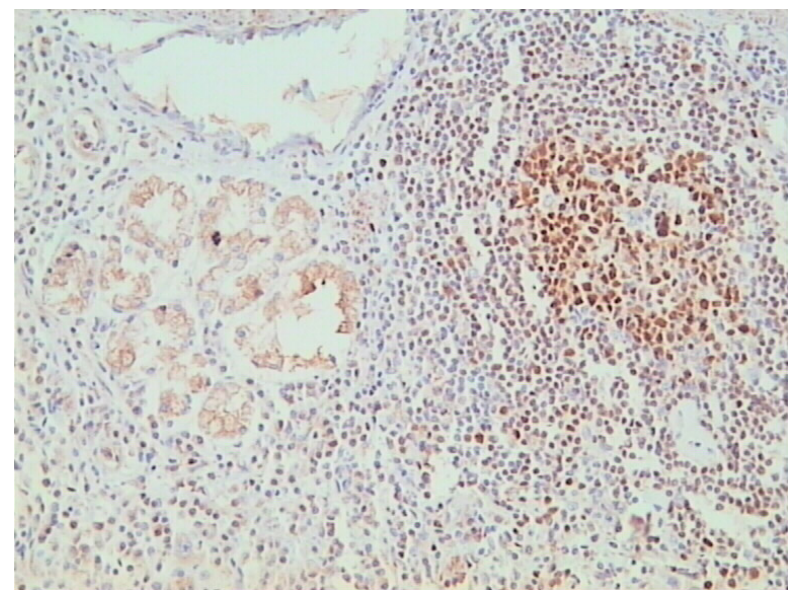

Figure 3. Nuclear staining on mucosal lymphoid tissue in gastric cancer but no nuclear staining in cancer cells.

by a single gene but the primary transcript can be spliced in a number of different ways. This alternative splicing is regulated differently in different cell types and that it may play an important role in the normal development 
of mammals.

Some experiments with high-density oligonucleotide microarray analysis with subsequent immunohistochemistry confirmation suggested that OCT-2 could be overexpressed in human gastric cancer with a significant statistical relationship with lymph node metastasis, but for the best of our knowledge no further studies exist on this subject.

OCT-2 transcription factor is also involved in the NF-KB cell signaling pathway which promotes cancer by inhibiting apoptosis of cancer cells and upregulates cytokines and growth factors which sustain cancer growth [6]. This cell signaling pathway is activated in chronic inflammation, a feature present in $H$. pylori infection of gastric mucosa, a recognized gastric carcinogenic agent.

OCT-1, another member of the POU homeodomain family of transcription factors that share with OCT-2 the ability to recognize the canonical octamer motif (ATGCAAAT) that regulates the transcription of various genes, is over expressed in intestinal metaplasia and intestinal type carcinomas in human gastric cancer and binds to (Caudal-related homeobox2) CDX2 in gastric cells, a gene that encodes an intestine-specific transcription factor crucial for the regulation of proliferation and differentiation of intestinal cells.

Also, it has been demonstrated that repression of two other transcription factors of the same family, OCT-3 and OCT-4, can induce differentiation of embryonic stem cells (ES) in trophectoderm (TE), an effect mimicked by over expression of CDX2 [7].

These observations prompted us to evaluate whether OCT-2 could in fact have a role in gastric oncogenesis and lymph node metastasis in human gastric cancer.

In this report we performed an immunohistochemistry assay on 69 archival paraffin blocks of gastric human tumors, obtained from patients undergoing gastrectomy in our Department of Surgery in Hospital Santa Maria, Lisboa, Portugal. All these gastric cancer cases are fully documented for correct pathological and clinical staging, histological classifications (WHO and Lauren) and follow-up equal or exciding 5 years. All were operated on with either a D1 or D2 lymph node dissection (16 to 25 lymph or 26 or more nodes harvested, respectively), and thus a correct lymph node staging was available according with the AJCC/UICC 2002 edition (Table 1).

Despite application and double check of the immunohistochemistry procedures we were not able to find any expression of OCT-2 transcription factor in any of our gastric cancer samples, on either type of tumor, gastric metaplasia or gastric normal mucosa. Although based exclusively on immunohistochemistry tests, the fact that only one report was found linking to OCT-2 and gastric cancer [5], combined with the absence of further publishing on this subject by the same group, makes us conclude that, based on immunohistochemistry methods, OCT-2 does not have a role in gastric oncogenesis and has no relationship with gastric cancer lymph node metastasis.

\section{References}

[1] Verrijser, C.P. and Van der Vliet, P.C. (1993) POU Domain Transcription Factors. Biochimica et Biophysica Acta, 1173, 1-21. http://dx.doi.org/10.1016/0167-4781(93)90237-8

[2] He, X., Treacy, M.N., Simmons, D.M., Ingraham, H.Á., Swanson, L.S. and Rosenfeld, M.G. (1989) Expression of a Large Family of POU-Domain Regulatory Genes in Mammalian Brain Development. Nature, 340, 35-42. http://dx.doi.org/10.1038/340035a0

[3] Corcoran, L.M., Karvelas, M., Nossal, G.J.V., Ye, Z.S., Jacks, T. and Baltimore, D. (1993) OCT-2 Although Not Required for Early B-Cell Development, Is Critical for Later B-Cell Maturation and for Postnatal Survival. Genes \& Develop, 7, 570-582. http://dx.doi.org/10.1101/gad.7.4.570

[4] Almeida, R., Almeida, J., Shoshkes, M., Mendes, N., Mesquita, P., Silva, E., Van Seuningen, I., Reis, C.A., Santos-Silva, F. and David, L. (2005) OCT-1 Is over Expressed in Intestinal Metaplasia and Intestinal Gastric Carcinomas and Binds to, But Does Not Transactivate, CDX2 in Gastric Cells. The Journal of Pathology, 207, 396-401. http://dx.doi.org/10.1002/path.1861

[5] Yoshitaka, H., Hirokazu, T., Tsutsumi, S., Machida, N., Chong, J., Fukayama, M., Kodama, T. and Aburatani, H. (2002) Global Gene Expression Analysis of Gastric Cancer by Oligonucleotide Microarrays. Cancer Research, 62, 233-240.

[6] Houghton, J.M., Morozov, A., Smirnova, I. and Wang, T.C. (2006) Stem Cells and Cancer. Seminars in Cancer Biology, 17, 191-203.

[7] Niwa, H., Toyooka, Y., Shimosato, D., Strumpf, D., Takahashi, K., Yagi, R. and Rossant, J. (2005) Interaction between OCT3/4 and Cdx2 Determines Trophoectoderm Differentiation. Cell, 123, 917-929.

http://dx.doi.org/10.1016/j.cell.2005.08.040 
Scientific Research Publishing (SCIRP) is one of the largest Open Access journal publishers. It is currently publishing more than 200 open access, online, peer-reviewed journals covering a wide range of academic disciplines. SCIRP serves the worldwide academic communities and contributes to the progress and application of science with its publication.

Other selected journals from SCIRP are listed as below. Submit your manuscript to us via either submit@scirp.org or Online Submission Portal.
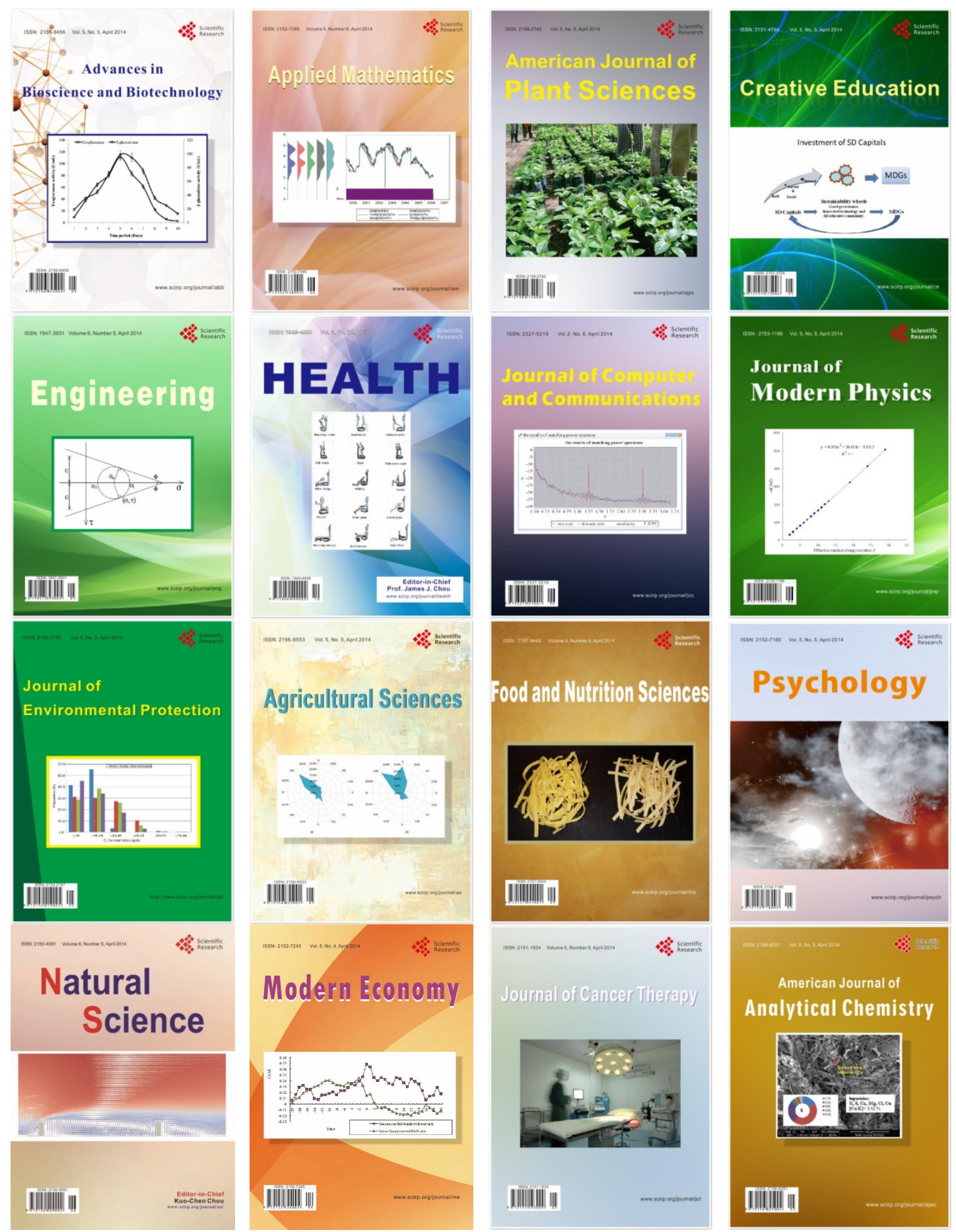